\title{
ZWEI BERGAMASCAS VOM ENDE DES 17. JAHRHUNDERTS
}

\author{
Jaroslav B u ž g a (Praha)
}

Der Name des seit dem 16. Jahrhundert bekannten Tanzes wird mit der norditalienischen Stadt Bergamo und auch mit "Bargue masque verknüpft. Die in ganz Europa verbreitete tänzerische Melodie machte sich auch in verschiedenen Gattungen der Kunstmusik, sogar in »Goldberg-Variationen « von Johann Sebastian Bach geltend. ${ }^{1}$

Als ein satirisches Lied und als cantus firmus einer Messe erschienen Bergamascas Ende des 17. Jahrhunderts auch in den Böhmischen Ländern. Der am berühmten Prämonstratenser Stift am Strahov in Prag wirkende Pater Jiř́ Evermodus Košetický schrieb um 1690 eine Bergamasca in seinen umfangreichen sechs grosse Bände umfassenden handschriftlichen Sammelband benannt »Quotlibeti...« nieder. ${ }^{2}$

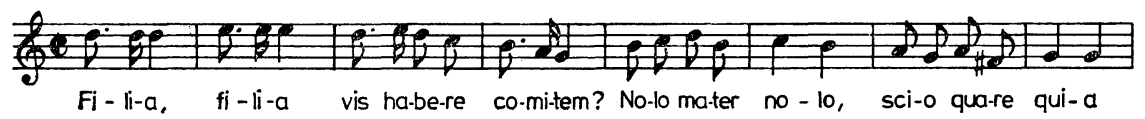

$\mathrm{Ob}$ Košetický den Text selbst verfasste ist unsicher. In seiner Handschrift befinden sich verschiedene tschechische, lateinische, deutsche kirchliche und weltliche, manche auch sozialkritische Liedertexte, mit eigenen Melodien, Sprüchen und Theaterstücken. Von der kirchlichen Zensur meisten untersagt und beschlaggenommen, zirkulierten

1 Vergl. Bergamasca, MGG, 1, Riemann Musik-Lexikon, Sachteil, Mainz 1967, Muzička enciklopedija 1, Zagreb 1971.

2 J. E. Košetický Quotlibeti..., Prag Strahovská knihovna (Strahover Bibliothek) Band 4, Sign. D G II, 7. Der Text hat insgesammt zehn Strophen. Die Mutter fragt die Tochter weiter, ob sie einen Adeligen (nobilem), Arzt (medicum), Musiker (musicum), Soldaten (militem), Pfarrer (Parochum), Mönch (monachium), Schuster (sutorem), Bauer (rusticum) haben möchte. Die Tochter lehnt alle ab und bejaht nur einen Studenten (studentem), oder Wein (vinum). Für den freundlichen Hinweis, dass es eine BergamascaMelodie ist, ist der Verfasser mit Dank dem verewigten Bencze Szabolcsi verpflichtet. 
sie heimlich unter der städtischen und ländlichen Bevölkerung. Ähnliche literarische Kompositionen und verschiedene Tanzmelodien erhielten sich auch in der Handschrift des Paters Christian Hirschmentzels aus dem Jahre 1692 in dem von Prag weit entfernten Zisterzienser Stift am Velehrad in Mähren. ${ }^{3}$ Die damalige Pflege der weltlichen Literatur, Musik und des Theaters bezeugen auch einige Berichte. Im Jahre 1651 luden Prager Minoriten den theaterliebenden Erzbischof Kardinal Harrach in ihr Kloster ein. Heimlich wollten sie ihm selbst eine »welsche Komödie« (also comedia dell arte) vorführen. Vor allem durften es nicht die Jesuiten erfahren. ${ }^{4}$

Die Bergamasca in Košetickýs Sammelband gehört zu den wenigen erhaltenen Denkmälern in mündlichen Überlieferungen aufblühender musikalischer und literarischer Gattungen. Ihre Melodie ist im 5.-8. Takt fast gleich mit dem 3.-4. Takt der Melodie der Bergamasca aus »Vilotte del Fiore $(1569) .5$ Wenn der punktierte Rhythmus und das schnelle Tempo (allabreve Takt) weggelassen werden, ist sie mit der ersten Hälfte der Bergamasca im quartären Takt aus »Vietoris Codex« (Slowakei um 1680) ${ }^{6}$ beinahe identisch und kann als ihre Variante aufgefasst werden.

Eine andere Bergamasca verwendete in der $\gg$ Missa sopra la bergamasca « der slowenische Komponist Janez Krstnik (Joannes Baptista) Dolar, dem der verehrte Jubilar mehrere Abhandlungen widmete. ${ }^{7}$ Der charakteristische tänzerische punktierte Rhythmus macht sich auch in dieser um zwei Takte kürzeren Melodie geltend. ${ }^{8}$ Volkommen wird der cantus firmus nur in den Trompeten-Stimmen im Kyrie, in den Gesangstimmen desselben Messe-Teiles schon verkürzt exponiert. Verhältnismässig wenig und grundsätzlich umgewandelt kommt er in Gloria, Credo (auf die Worte »resurrectionem expecto«), Benedictus (»in nomine«) und Agnus (»dona nobis pacem«) vor.

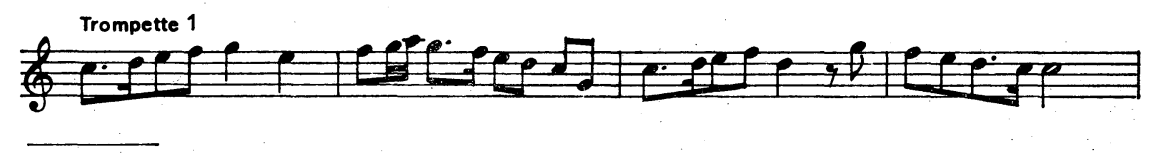

3 Vergl. J. Bužga, Die Volksmusik im Zeitalter des Barocks' in Böhmischen Ländern, Kongressbericht Berkeley 1977, Kassel - Basel 1981.

${ }_{4}$ F. Menčík, Př́spěvky $k$ dějinám českého divadla (Beiträge zur Geschichte des tschechischen Theaters), Prag 1895.

5 Vergl. Bergamasca, Riemann Musiklexikon.

6 Vergl. B. Szabolcsi, Probleme der alten ungarischen Musikgeschichte, Zeitschrift für Musikwissenschaft VIII, 1925-26, L. Burlas, J. Fišer, A. Hořejš, Hudba na Slovensku $v$ XVII. storočí (Musik in der Slowakei im 17. Jahrhundert), Bratislava 1954.

7 D. Cvetko, Joannes Baptista Dolar, (tschechisch), Hudební věda 1966, ders., Dolar, MGG 15 (Supplement).

8 Joannes Baptista Tolar (Dolar), Missa sopra la bergamasca von der Musiksammlung in Kroměříž spartierte E. Trolda, Prag, Národní muzeum, hudební oddělení (Musikabteilung des Nationalmuseums in Prag), Sign: XXVIII E 154, vergl. A. Buchner, Hudební sbírka E. Troldy, Prag 1954. 
Die Messe wurde in der berühmten fürstbischöflichen Kapelle in Kroměříž (Kremsier) in Mähren, die Heinrich Biber (bis 1670) und nach ihm Pavel Josef Vejvanovský (bis 1693) leiteten, aufgeführt. ${ }^{9}$ Über direkte Beziehungen Dolars zur Kapelle ist nichts näheres bekannt. Mehrere in Kroměříž erhaltene Abschriften seiner Kompositionen sind mit dem Namen Tolar (die tschechische Bezeichnung für Thaler) versehen. Irrtümlich wurde er so für einen unbekannten tschechischen Komponisten gehalten. Verschiedene seine Kompositionen sind auch in den Musikinventarien von dem Piaristen-Kollegium in Slaný und von dem Zisterzienser Stift in Ossek (Osseg) in Böhmen notiert. ${ }^{10}$ Ähnlich wie andere in Kroměříž aufbewahrte Kompositionen wurde die Messe vermutlich in Wien erworben, wo Dolar als Kirchenmusiker wirkte und die damals beliebte Bergamasca kennen lernen konnte. ${ }^{11}$ Die auch von anderen Komponisten benützte Parodie-Technik musste Dolar, der noch eine "Missa Vilana « schrieb, besonders vertraut sein. ${ }^{12}$ In Kroměřiž erhielten sich auch weitere verschiedene Parodie-Messen aus der zweiten Hälfte des 17. Jahrhunderts. ${ }^{13}$ Der Salzburger Domkapellmeister Andreas Hofer schrieb die »Missa, Quod vobis videtur, « ein anonymer Komponist die nur in Bruchstücken erhaltene "Missa super, Non habemus vinum. "Der tschechische Komponist Adam Michna $z$ Otradovic benüzte als cantus firmus das Weinachtslied »Již slunce $z$ hvězdy vyšlo« (Die Sonne ist aus dem Stern bereits aufgegangen). ${ }^{14}$ Der sonst unbekannte Priester Reverendissimus Dominus Maximilianus Kodras verwendete in "Missa di polaka " eine Polonaise. ${ }^{15}$ Dieser damals beliebte Tanz kommt auch im erwähnten »Codex Vietoris« vor und wird auch als »Chorea Polonica in den Synopsen der Schuldramen genannt. ${ }^{16}$

Frei ansetzende, mit klangvollen continuo begleitete vokale und instrumentale Stimmen in Dolars Messe folgen nicht mehr strengen in alter Polyphonie geltenden Regeln. Die verwandelte Kompositionstech-

9 Über die Kapelle in Kroměříž (Kremsier) vergl.: A. Breitenbacher, Hudební sbírka kolegiátního kostela sv. Moŕice $v$ Kroměŕízi (Die Musiksammlung der Kollegiatkirche in Kremsier), Olomouc 1928, E. H. Meyer, Die Bedeutung der Instrumentalmusik am fürstbischöflichen Hofe zu Olomouc (Olmütz) in Kroměříž (Kremsier), MGG 7, J. Sehnal, Die Musikpflege des Olmützer Bischof Karl Lichtenstein-Castelcorn in Kremsier, Kirchenmusikalisches Jahrbuch 1967.

10 Jan Krrtitel Tolar, Balleti e sonate (J. Pohanka), Musica antiqua Bohemica, Band 40, Prag 1959.

11 Vergl. Dolar, MGG 15, Bergamasca MGG 1.

12 A. Breitenbacher, Hudbení sbírka.

13 Ebenda.

14 Vergl. J. Bužga, Der tschechische Barockkomponist Adam Michna $z$ Otradovic (um 1600-1676), Festschrift Heinrich Besseler, Leipzig 1961.

15 A. Breitenbacher, ebenda.

16 Vergl. Polonaise, MGG 10, Riemann Musiklexikon, L. Burlas usw. Hudba na Slovensku. Verschiedene Polonaisen bringen Žródla do historii muzyki polskij (Quellen zur Geschichte der polnischen Musik; Redaktion Z. Szweykowski), Warschau, Band I, II, VI, IX, XIII, XVII, XIX, XXI. 
nik unterscheidet sich auch von dem im Zeitalter des Hochbarocks üblichen Parodie-Verfahren. In Werken von J. S. Bach wurden ganze Kompositionen mit ursprünglich weltlichen Texten mit neuen liturgischen Texten versehen und zu kirchlichen Zwecken umgewandelt.17

Die tänzerischen Melodien in der Messe von Dolar und von anderen Komponisten werfen die Frage auf, ob sie die Zeitgenossen als weltliche empfanden, besonders im Gegensatz zum Choral mit dem sie bei den Gottesdiensten unmittelbar konfrontiert wurden.

Weltliche Melodien verwendeten schon im 16. Jahrhundert die Böhmischen Brüder in ihren Kantionalien. Ob dabei tatsächlich ein schroffer Gegensatz zwischen dem Profanen und Religiösen allgemein aufgefasst wurde, ist kaum denkbar. Bei den strengen Sitten und der Frömigkeit der Brüder lässt sich eher voraussetzen, dass sie den Melodien keine besondere Aufmerksamkeit schenkten. Ihre ursprüngliche Verwendung berücksichtigten sie kaum. Für ihren Erzfeind den gelehrten Jesuiten Václav Šturm war es aber ein willkommener Anlass zur heftigen Kritik ihres Kantionales und zu Vorwürfen, dass er einige dort benüzte Melodien als Jüngling in »Schänkhäusern hörte. «18 $\mathrm{Im} 17$. Jahrhundert wurden Kirchenlieder geläufig in Deutschland ${ }^{19}$ und in Böhmischen Ländern auf tänzerische Melodien gesungen, ${ }^{20}$ ohne dass es Anstoss erweckte, oder Ablehnung hervorrief.

Nach dem jesuitischen »Ad Maiorem Dei Gloriam « - Dolar selbst war Jesuit - waren alle greifbaren die menschlichen Sinne anreizenden Mittel den religiösen Zwecken zugewandt. Die musikalischen Veranstaltungen bei Gottesdiensten im Zeitalter der Gegenreformation lockten besonders äussere Pracht liebende adelige Gönner an. Ähnlich wie das Ordenstheater führten sie auch zu verschiedenen Kompetenz-Streitigkeiten zwischen den kirchlichen Institutionen. Die oft zu einer Aufführung bestimmten Kompositionen wurden nur undiferenziert als Klangbegleitung der liturgischen Zeremonien, als "Umgangsmusik " in Besselers Sinne wahrgenommen. ${ }^{21}$ Das neuzeitliche historische Interesse schäzt sie ähnlich wie andere damalige Gebrauchsgegenstände als Kunstwerke.

Die Darstellung der zwei Bergamascas zeigt die Schichtung der musikalischen Gattungen am Ende des 17. Jahrhunderts in den Böhmischen Ländern und die Verbreitung bekannter tänzerischer Melolexikon.

17 Vergl. Parodie und Kontrafaktur, MGG 10, Parodie, Riemann Musik-

18 Vergl. J. Bužga, Zur musikalischen Problematik der alttschechischen Kantionalien, Die Musikforschung XII, 1959.

19 E. H. Meyer, Instrumentale Tanzkompositionen und Volksmusik in Deutschland um 1660 (E. H. Meyer, Musik der Renaissance-Aufklärung-Klassik, Leipzig 1979).

20 Vergl. J. Bužga, Der tschechische Barockkomponist.

21 Vergl. H. Besseler, Umgangsmusik und Darbietungsmusik im 16. Jahrhundert, (H. Besseler, Aufsätze zur Musikästhetik und Musekgeschichte, Leipzig 1978). 
dien. Zugleich trägt sie zur Erleuterung der Problematik der ParodieKompositionstechnik und der weltlichen Elemente in der Kirchenmusik bei.

\section{POVZETEK}

Dve različni melodiji bergamasce $s$ konca 17. stoletja kažeta, da so bile melodije tega plesa znane tudi $\mathrm{v}$ čeških deželah. Melodija iz Košetickega zbornika (Praga okrog 1690, primer 1) s parodističnim latinskim tekstom je ritmično spremenjena varianta dela melodije bergamasce iz Vietoris Codexa (Slovaška okrog 1680). Podobno ritmizirano melodijo je uporabil slovenski skladatelj Dolar kot cantus firmus v skladbi Missa sopra la bergamasca (primer 2), ki so jo izvedli $\mathrm{v}$ znameniti knezoškofijski kapeli $\mathrm{v}$ Kroměř́̌žu. Dolarjeva maša dokumentira tedanjo specifično tehniko parodiranja, ki se razlikuje od podobne kompozicijske tehnike obdobja vokalne polifonije kakor tudi poznega baroka (J. S. Bacha). Parodična maša tudi sproži vprašanje o posvetnih elementih, ki so bili v cerkveni glasbi protireformacije običajni. Očitno so gledali na cerkvene kompozicije kot na spremljavo ceremonij, kot na nekakšno "Umgangsmusik" v Besselerjevem smislu in jih niso imeli za umetnine $\mathrm{v}$ današnjem pomenu besede. 